\title{
Non-invasive measurement of venous oxygen saturation as early marker of impaired oxygen supply in preterm neonates
}

\author{
Lukas P. Mileder, Berndt Urlesberger, Julia Buchmayer, Nariae Baik-Schneditz, Bernhard Schwaberger, \\ Nina Höller, Gerhard Pichler
}

Research Unit for Neonatal Micro- and Macrocirculation, Department of Paediatrics and Adolescent Medicine, Medical University of Graz, Graz, Austria

Division of Neonatology, Department of Paediatrics and Adolescent Medicine, Medical University of Graz, Graz, Austria

\section{Background and aims}

Venous oxygen saturation $\left(\mathrm{SvO}_{2}\right)$ decreases in situations of impaired oxygen supply of tissues, even if there is no reduction in arterial oxygen saturation $\left(\mathrm{SaO}_{2}\right)$. Andersen et al. (Pediatrics 2017) suggested that "measuring oxygen extraction or the venous oxygen reservoir could define a physiologically based definition of adequate oxygen(-ation)". Hence, our aim was to assess whether there is a difference in peripheral muscle $\mathrm{SvO}_{2}\left(\mathrm{pSvO}_{2}\right)$ and fractional oxygen extraction (pFOE) measured by near-infrared spectroscopy (NIRS) between preterm neonates with an early infection compared to those without an early infection during the first 36 hours of life.

\section{Methods}

We analysed secondary outcome parameters of a randomized controlled trial („Avoiding Hypotension in Preterm Neonates": Pichler et al. Front Pediatr 2018) and included preterm neonates at risk of early infection, in whom NIRS measurement including peripheral venous occlusion was performed. Infection was diagnosed by clinical signs and a positive blood culture and/or C-reactive protein ( $\mathrm{CrP})$ above the cut-off value (10mg/l).

We measured peripheral muscle tissue oxygenation index (pTOI) using NIRO 200-NX (Hamamatsu Photonics, Japan), calculated pFOE from pTOI and $\mathrm{SaO}_{2}$ and measured $\mathrm{pSvO}_{2}$ via venous occlusion method. For this, a blood pressure cuff was placed around the upper arm or thigh and inflated to a pressure level above the estimated venous blood pressure, thus interrupting venous outflow but leaving arterial inflow undisturbed (Figure 1).

\section{Results}

We included 33 preterm neonates with a mean gestational age of $33.2 \pm 1.5$ weeks (birth weight: $2007 \pm 486 \mathrm{~g} ; \mathrm{m}: \mathrm{w}=20: 13$ ). NIRS measurements and venous occlusions were performed at a median of 16.0 hours (1.0-28.0) after birth.

Of the 33 preterm neonates, five (15.2\%) developed an infection (mean leucocytes: $17.252 \pm 11.129 / \mu \mathrm{l}$, mean CrP: $6.7 \pm$ $4.4 \mathrm{mg} / \mathrm{l})$. Peripheral $\mathrm{SvO}_{2}$ was significantly lower (49.3\% versus $59.0 \%$; $p=0.018)$ and pFOE significantly higher (47.8 versus 38.8; $p=0.026)$ in preterm neonates with an infection (Figures 2a \& 2b).

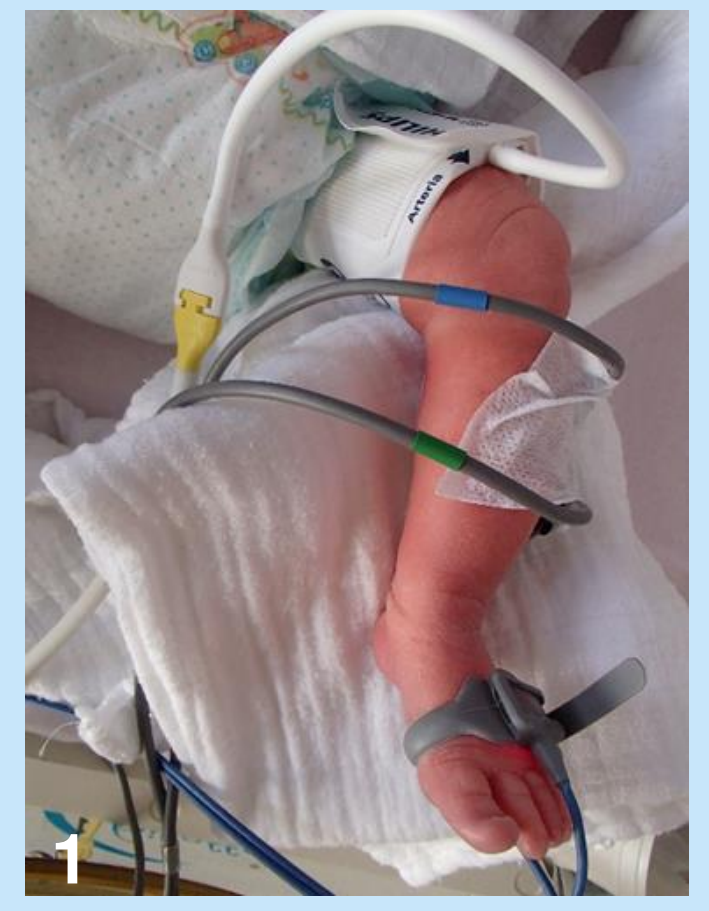

Figure 1: NIRSmeasurement with venous occlusion (thigh), NIRS sensors (calf) and pulse oximetry (foot).

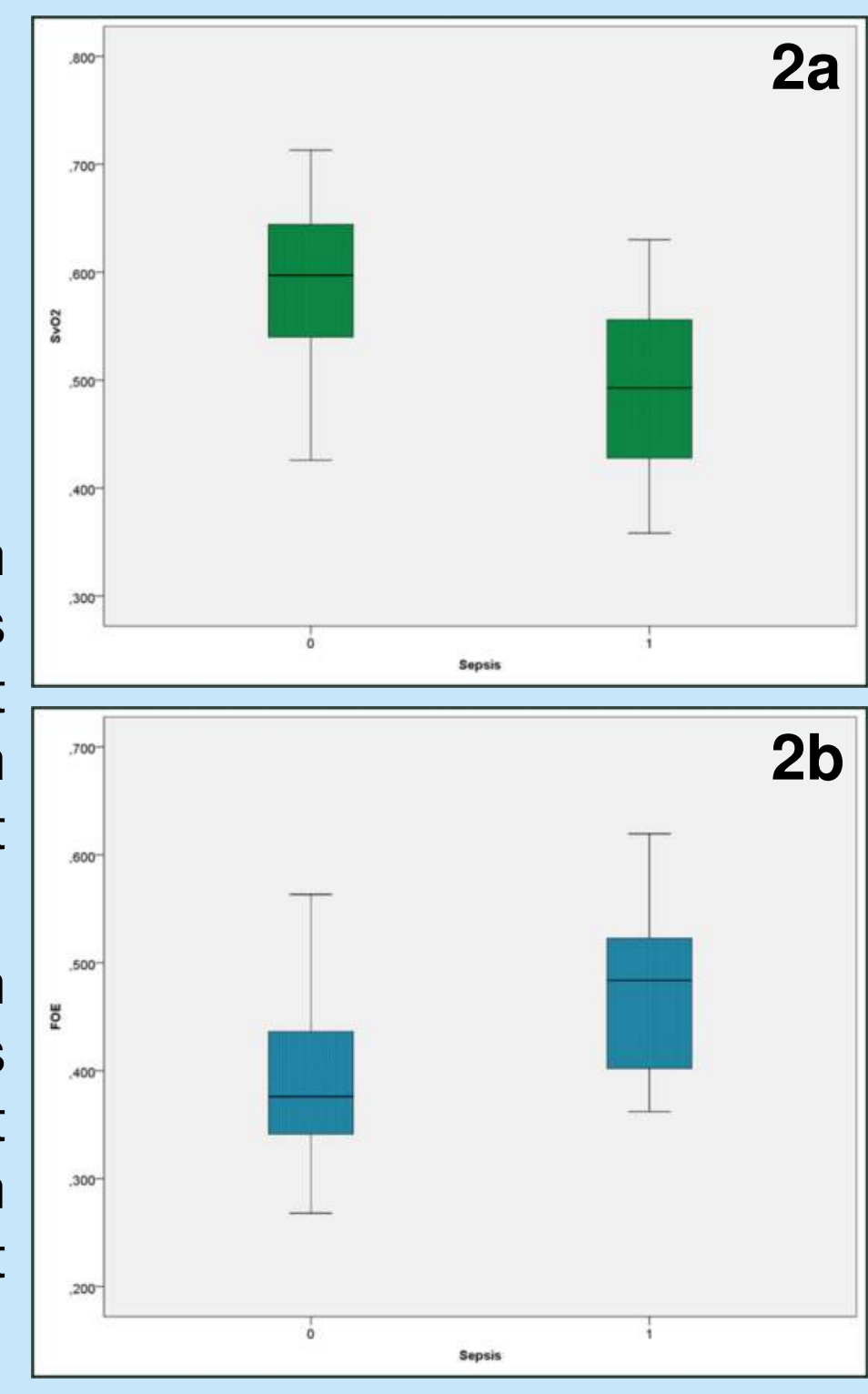

\section{Conclusions}

We found impaired $\mathrm{pSvO}_{2}$ and increased pFOE in preterm neonates with an early infection. Noninvasive measurement of these parameters is feasible and could be utilized for early detection of impaired peripheral oxygenation and (micro-)circulation in the future. 